\title{
Static local-field factors in a two-dimensional electron liquid
}

\author{
R. Asgari, ${ }^{1}$ A. L. Subaş $1,{ }^{2}$ A. A. Sabouri-Dodaran, ${ }^{1,3}$ and B. Tanatar ${ }^{2}$ \\ ${ }^{1}$ Institute for Studies in Theoretical Physics and Mathematics, Tehran 19395-5531, Iran \\ ${ }^{2}$ Department of Physics, Bilkent University, 06800 Bilkent, Ankara, Turkey \\ ${ }^{3}$ Payame Noor University, Tehran 19395-4697, Iran
}

(Received 23 January 2006; revised manuscript received 10 July 2006; published 23 October 2006)

\begin{abstract}
We present a numerical calculation for the static spin-symmetric and spin-antisymmetric local-field factors as a function of density in a two-dimensional (2D) unpolarized electron liquid. We use a recent analytical expression for the spin-resolved pair distribution function of a $2 \mathrm{D}$ electron liquid based on quantum Monte Carlo simulation data and accurate correlation energy as input to construct the local-field factors from the fluctuation-dissipation theorem. We obtain good agreement with data from quantum Monte Carlo studies of the $2 \mathrm{D}$ electron liquid over an extensive range of density.
\end{abstract}

DOI: $10.1103 /$ PhysRevB.74.155319

PACS number(s): 71.10.Ca, 05.30.Fk, 71.30.+h

\section{INTRODUCTION}

The model of a two-dimensional (2D) electron liquid, that is, electrons confined to a plane, interacting with each other via the $1 / r$ Coulomb potential in a neutralizing background is very useful in understanding properties of physical systems realized in semiconducting structures. ${ }^{1}$ Advances in fabrication techniques are making it possible to form samples of low-dimensional electron systems of high quality and of very low density, thus allowing comparison between experiment and theory. Because of this, there has been a large amount of experimental and theoretical work in recent years on 2D electron systems, some of which are motivated by the observation of metal-insulator transition ${ }^{2}$ and possible spintronics or nanotechnology applications.

The most reliable results on the ground-state properties of homogeneous electron gas are provided by quantum Monte Carlo (QMC) simulations. ${ }^{3-6}$ Here, not only the accurate ground-state energies and static correlation functions are calculated $^{3,4,6}$ but also the static response functions are obtained $^{5}$ using novel algorithms. These results are very valuable as they probe the highly correlated regime at very low density which has become accessible in recent experiments.

Traditionally, the correlation effects are treated within a variety of many-body techniques. The cornerstone of the theoretical formulation of the dielectric properties is the localfield factor embodying such effects. ${ }^{1,7}$ A popular approach is the self-consistent field scheme of Singwi et al. ${ }^{8}$ (STLS) and its various variants ${ }^{9}$ which have been used in many an application. The importance of having full knowledge of localfield factors is also revealed through their connection to the exchange-correlation kernels used in density functional theory based calculations for inhomogeneous systems and in studies of quasiparticle properties in the electron Fermi liquid. ${ }^{10}$

Although there has been some recent progress in developing wave vector and frequency dependent local-field factors ${ }^{11,12}$ in most of the applications still the frequency independent, static local-field factors are used. A natural line of investigation is to combine what is known from QMC calculations and the exact limiting forms afforded by various sum- rules to construct practical local-field factors to be used in the evaluation of further physical quantities..$^{13-15}$

The purpose of this work is to employ the available information on the static structure factors from QMC simulations to extract the $\omega$-independent local-field corrections via the fluctuation-dissipation theorem. In fact, the idea here is not entirely new, it has been used by Iwamoto et al. ${ }^{16}$ in the context of 3D electron liquid. Presently, there exist simulation data of the static structure factor ${ }^{3,6}$ for $2 \mathrm{D}$ electron liquid and static response ${ }^{5}$ results with which we can compare our findings. There has been various other efforts to construct local-field factors from the QMC structure factors utilizing different closure relations. ${ }^{17,18}$ Some of these such as the STLS approximation involve integral relations based on approximate theories, thus their success is somewhat limited because of the underlying assumptions in the closure relations used. We find that our fluctuation-dissipation theorem based procedure yields good agreement with the QMC localfield factors both in the density and spin-density channels.

In the following we first outline the model and theoretical framework with which we construct the static local-field factors of a 2D electron system. We next present our results for illustrative cases at different densities. We discuss the results of our calculations also in view of other theoretical approaches. We conclude with a brief summary.

\section{MODEL AND THEORY}

We consider a 2D electron system interacting via the long range Coulomb interaction whose Fourier transform is $V(q)=2 \pi e^{2} /\left(\epsilon_{0} q\right)$ where $\epsilon_{0}$ is the background dielectric constant. The system is characterized by the dimensionless interaction strength $r_{s}=1 /\left(\pi n a_{B}^{* 2}\right)^{1 / 2}$, where $n$ is the $2 \mathrm{D}$ electron density and $a_{B}^{*}=\hbar^{2} \epsilon_{0} /\left(m e^{2}\right)$ is the effective Bohr radius defined in terms of the band mass $m$ of electrons in the semiconductor structure. The Fermi wave vector $k_{F}$ is related to the density by $k_{F}=\sqrt{2 \pi n}$.

The dielectric properties of electron liquids are described by the density-density response function

$$
\chi_{s}(q, \omega)=\frac{\chi_{0}(q, \omega)}{1-V(q)\left[1-G_{s}(q, \omega)\right] \chi_{0}(q, \omega)},
$$

and spin-spin response function 


$$
\chi_{a}(q, \omega)=\frac{\chi_{0}(q, \omega)}{1+V(q) G_{a}(q, \omega) \chi_{0}(q, \omega)},
$$

where $\chi_{0}(q, \omega)$ is the noninteracting response function, i.e., Lindhard function which contains the noninteracting occupation numbers. The wave vector and frequency dependent local-field factors $G_{s}(q, \omega)$ and $G_{a}(q, \omega)$ describe the shortrange electron correlations in the spin-symmetric and spinantisymmetric channels. In this work, we approximate the dynamic local-field factors by their $\omega$-independent, $G_{s, a}(q)$ to obtain only the wave vector dependence. The frequency dependence of $G_{s, a}(q, \omega)$ has recently been considered by Atwal et al. ${ }^{11}$ and Qian and Vignale. ${ }^{12}$

In the following sections we first introduce the polarization potential theory as developed by Iwamoto ${ }^{15}$ for $2 \mathrm{D}$ electron gas and then outline our approach based on the fluctuation-dissipation theorem to extract local-field factors from QMC static structure factors.

\section{A. Polarization potential}

In this approach configuration and momentum-space pseudopotentials are introduced to describe the short-range density and spin-density correlations which are in turn related to the local-field factors. ${ }^{15,19}$ The spin-symmetric and spin-antisymmetric local-field factors are modeled in a fashion similar to the Hubbard approximation to the local-field factors as

$$
\begin{aligned}
G_{s}(q) & =\frac{1}{2}\left[G_{\uparrow \uparrow}(q)+G_{\uparrow \downarrow}(q)\right] \\
& =\frac{1}{2}\left[\frac{1}{\left(q^{2}+q_{\uparrow \uparrow}^{2}\right)^{1 / 2}}+\frac{q}{\left(q^{2}+q_{\uparrow \downarrow}^{2}\right)^{1 / 2}}\right],
\end{aligned}
$$

and

$$
\begin{aligned}
G_{a}(q) & =\frac{1}{2}\left[G_{\uparrow \uparrow}(q)-G_{\uparrow \downarrow}(q)\right] \\
& =\frac{1}{2}\left[\frac{1}{\left(q^{2}+q_{\uparrow \uparrow}^{2}\right)^{1 / 2}}-\frac{q}{\left(q^{2}+q_{\uparrow \downarrow}^{2}\right)^{1 / 2}}\right],
\end{aligned}
$$

where $q_{\uparrow \uparrow}$ and $q_{\uparrow \downarrow}$ are two parameters to be determined for each value of $r_{s}$. They are obtained using the compressibility and spin-susceptibility sum rules which relate to the longwavelength behavior of $G_{s}(q)$ and $G_{a}(q)$, respectively,

$$
\frac{\kappa_{0}}{\kappa}-1=-\frac{r_{s}}{\sqrt{2}}\left(\frac{k_{F}}{q_{\uparrow \uparrow}}+\frac{k_{F}}{q_{\uparrow \downarrow}}\right),
$$

and

$$
\frac{\chi_{P}^{0}}{\chi_{P}}-1=-\frac{r_{s}}{\sqrt{2}}\left(\frac{k_{F}}{q_{\uparrow \uparrow}}-\frac{k_{F}}{q_{\uparrow \downarrow}}\right) .
$$

Here $\kappa$ and $\chi_{P}$ are the compressibility and Pauli spinsusceptibility of the 2D electron liquid, and $\kappa_{0}=\pi r_{s}^{4} / 2$ and $\chi_{P}^{0}=\mu_{B}^{2} m / \pi \hbar^{2}$, where $\mu_{B}$ is the Bohr magneton, are their corresponding noninteracting values. These thermodynamic quantities are related to the correlation energy $\epsilon_{c}$ by

$$
\frac{\kappa_{0}}{\kappa}=1-\frac{\sqrt{2}}{\pi} r_{s}-\frac{r_{s}^{3}}{8} \frac{\partial \epsilon_{c}}{\partial r_{s}}+\frac{r_{s}^{4}}{8} \frac{\partial^{2} \epsilon_{c}}{\partial r_{s}^{2}}
$$

and

$$
\frac{\chi_{P}^{0}}{\chi_{P}}=1-\frac{\sqrt{2}}{\pi} r_{s}+\left.\frac{r_{s}^{2}}{2} \frac{\partial^{2} \epsilon_{c}}{\partial \xi^{2}}\right|_{\xi=0},
$$

where $\xi=\left(N_{\uparrow}-N_{\downarrow}\right) / N$ is the degree of spin-polarization. We use the parametrized form of $\epsilon_{c}$ from the recent QMC simulations of Attaccalite et $a l_{.}{ }^{4}$ to find the parameters $q_{\uparrow}$ and $q_{\downarrow}$ and thereby construct $G_{s}(q)$ and $G_{a}(q)$ within the polarization potential theory. Note that the polarization potential theory makes use of the QMC ground-state energy (or correlation energy) to only fix the correct long-wavelength behavior of $G_{s}(q)$ and $G_{a}(q)$. The rest of the $q$ behavior is modeled by the Hubbard-like approximation given above.

\section{B. Fluctuation-dissipation theorem}

The fluctuation-dissipation theorem which is of paramount importance for systems in equilibrium relates the dynamic susceptibilities defined above to the static structure factors

$$
S_{s, a}(q)=-\frac{1}{n \pi} \int_{0}^{\infty} d \omega \Im\left[\chi_{s, a}(q, \omega)\right] .
$$

Within the assumption of static local-field factors, as $\chi_{s}(q, \omega)$ and $\chi_{a}(q, \omega)$ depend on $G_{s}(q)$ and $G_{a}(q)$, respectively, the above integral expression allows one to determine the localfield factors once the static structure factors are given. In a more general setting with frequency dependent local-field factors $G_{s, a}(q, \omega)$ to utilize the fluctuation-dissipation theorem one should have a model for the frequency dependence. This evidently is a more difficult problem which is beyond our present scope. We, thus, assume static local-field factors from the outset, and try to correct its limitations as discussed below. The static structure factors for a $2 \mathrm{D}$ electron liquid is known to a high accuracy from QMC simulations of GoriGiorgi et al. ${ }^{6}$ which we use in this work.

As an additional simplification to the above procedure, one can further approximate the full $\chi_{0}(q, \omega)$ by the meanspherical approximation (MSA), viz.

$$
\chi_{0}^{\mathrm{MSA}}(q, \omega)=\frac{\left(n \hbar^{2} q^{2} / m\right)}{(\omega+i \eta)^{2}-\left[\frac{\hbar^{2} q^{2}}{2 m S_{0}(q)}\right]^{2}}
$$

in which $S_{0}(q)$ is the static structure factor of the noninteracting system and $\eta$ is a positive infinitesimal quantity. With this approximation, the fluctuation-dissipation integral can be performed analytically to yield

$$
G_{s}(q)=1-\frac{\sqrt{2}}{4 r_{s}}\left(\frac{q}{k_{F}}\right)^{3}\left[\frac{1}{S_{s}(q)^{2}}-\frac{1}{S_{0}(q)^{2}}\right],
$$

and 


$$
G_{a}(q)=-\frac{\sqrt{2}}{4 r_{s}}\left(\frac{q}{k_{F}}\right)^{3}\left[\frac{1}{S_{a}(q)^{2}}-\frac{1}{S_{0}(q)^{2}}\right] .
$$

Expressions similar to the above have been used in the context of charged boson fluids ${ }^{20}$ with the replacement of $S_{0}(q)$ by unity. The MSA is essentially a plasmon-pole type approximation, where the particle-hole excitations are replaced by a single collective mode. As will be shown shortly, it works quite well when the frequency integral in the fluctuation-dissipation theorem is performed.

\section{RESULTS AND DISCUSSION}

We have calculated the spin-symmetric $G_{s}(q)$ and spinantisymmetric $G_{a}(q)$ static local-field factors for a paramagnetic 2D electron liquid in different approaches. First, we have used the polarization potential approach of Iwamoto ${ }^{15}$ supplemented with the recent QMC correlation energy of Attaccalite et $a l .{ }^{4}$ The results are very close to the earlier calculations by Iwamoto $^{15}$ who used the Tanatar and Ceperley ${ }^{3}$ QMC correlation energy. This is quite understandable since both QMC calculations yield the same correlation energy for a paramagnetic electron liquid. Next, we have calculated the static local-field factors by solving Eq. (9) for $G_{s}(q)$ and $G_{a}(q)$, given the static structure factors $S_{s}(q)$ and $S_{a}(q)$. For this purpose, we have first calculated the spinsymmetric and spin-antisymmetric pair distribution functions from the QMC based analytical expressions ${ }^{6}$ and performed the Fourier transform

$$
S_{s, a}(q)=1+n \int d \mathbf{r}\left[g_{s, a}(r)-1\right] \exp (-i \mathbf{q} \cdot \mathbf{r}) .
$$

These $S_{s, a}(q)$ are assured to satisfy the plasmon and $f$-sum rules. Finally, the local-field factors within the MSA are calculated from the analytic expressions above using the same QMC static structure factors.

Before presenting our numerical calculations, we outline a different way to formulate the response functions of an electron gas, based on employing the modified form of the Lindhard function which uses the exact occupation numbers in Eqs. (1) and (2). The density and spin-density response functions are now expressed as (assuming frequency independent local-field corrections)

$$
\chi_{s}(q, \omega)=\frac{\bar{\chi}_{0}(q, \omega)}{1-V(q)\left[1-\bar{G}_{s}(q)\right] \bar{\chi}_{0}(q, \omega)},
$$

and

$$
\chi_{a}(q, \omega)=\frac{\bar{\chi}_{0}(q, \omega)}{1+V(q) \bar{G}_{a}(q) \bar{\chi}_{0}(q, \omega)},
$$

where $\bar{\chi}_{0}(q, \omega)$ is the modified form of the Lindhard function. Comparing Eqs. (14) and (15) with Eqs. (1) and (2), we have

$$
G_{s, a}(q)=\bar{G}_{s, a}(q)+\frac{1}{V(q)}\left[\frac{1}{\bar{\chi}_{0}(q, 0)}-\frac{1}{\chi_{0}(q, 0)}\right] .
$$

This approach leads one to define ${ }^{11}$ a further local-field factor $G_{n}(q)$ associated with occupation number renormaliza-
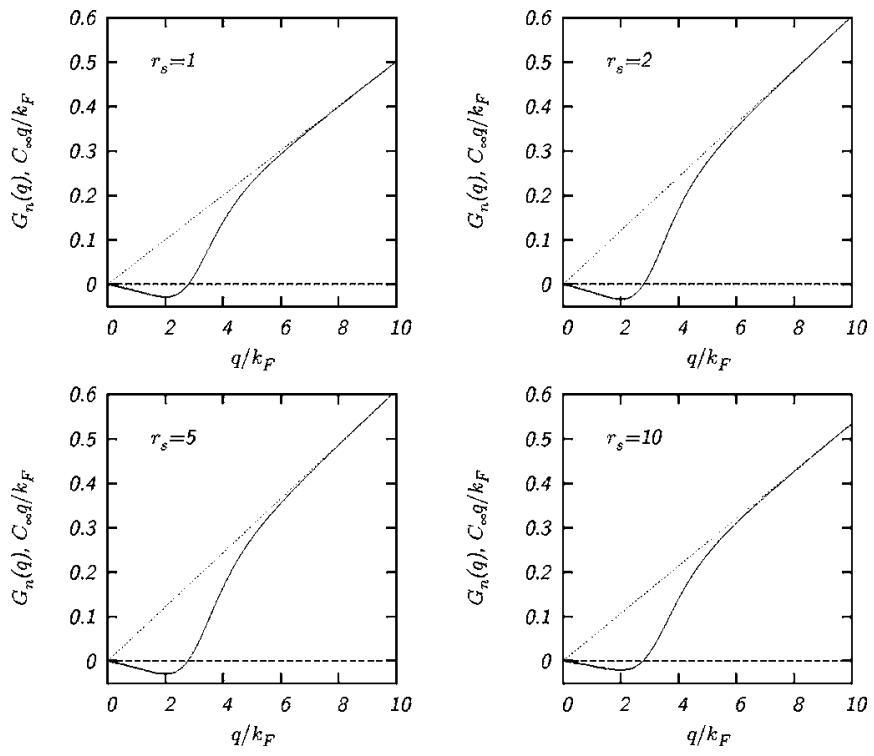

FIG. 1. The local-field factor $G_{n}(q)$ (solid lines) for various values of $r_{s}$ as computed according to Ref. 11, in comparison with its linear asymptotic curve (dotted lines), i.e., $C_{\infty}\left(q / k_{F}\right)$.

tion of $\chi_{0}(q, \omega)$. In this case the modified many-body localfield factors are given by $\bar{G}_{s, a}=G_{s, a}(q)+G_{n}(q)$ where

$$
G_{n}(q)=-\frac{1}{V(q)}\left[\frac{1}{\bar{\chi}_{0}(q, 0)}-\frac{1}{\chi_{0}(q, 0)}\right] .
$$

In comparing our local-field factors with $G_{s}^{\mathrm{QMC}}(q)$ which fundamentally take into account this effect, we subtract the $G_{n}(q)$ from QMC to render the comparison on the same footing as also discussed by Dharma-wardana and Perrot $^{21}$ and Atwal et al. ${ }^{11}$ Atwal et al. ${ }^{11}$ constructed the $G_{n}(q)$ for a 2D electron gas by considering the first-order correction to the proper polarizability function and further they used an approximate expression based on the perturbation calculations for the ratio of the gradients of the respective local-field factors at $q=0$ for $1 \leqslant r_{s} \leqslant 10$ in Eq. (22) in their paper. ${ }^{22}$ All these approximations increase inaccuracy in physical quantities at higher $r_{s}$ when their $G_{n}(q)$ is used and hence we expect larger deviation between our numerically obtained results and accurate QMC data in this regime. The main results of our work are shown in Figs. 1-5.

In Fig. $1 G_{n}(q)$ and its asymptotic behavior (extended to all $q$ values) are shown for $r_{s}=1,2,5$, and 10 . As it is clear from this figure, $G_{n}(q)$ has a structure for $q \leqslant 4 k_{F}$. It yields negative values in the long-wavelength limit and becomes positive around $q \approx 3 k_{F}$. The $G_{n}(q)$ tends to the asymptotic linear curve at $q \cong 8 k_{F}$. Note that the asymptotic behavior of $G_{n}(q)$ at large $q$ is $C_{\infty} q / k_{F}$, where $C_{\infty}=-\left(r_{s} / 2 \sqrt{2}\right) \frac{d}{d r_{s}}\left(r_{s} \epsilon_{c}\right)$ is related to the difference between interacting and noninteracting kinetic energies. ${ }^{23}$

In Fig. 2 we show our calculated spin-symmetric localfield factor $G_{s}(q)$ for $r_{s}=1,2,5$, and 10. Also shown are the $G_{s}(q)$ values calculated within the Iwamoto's polarization potential approach and by the fluctuation-dissipation proce- 

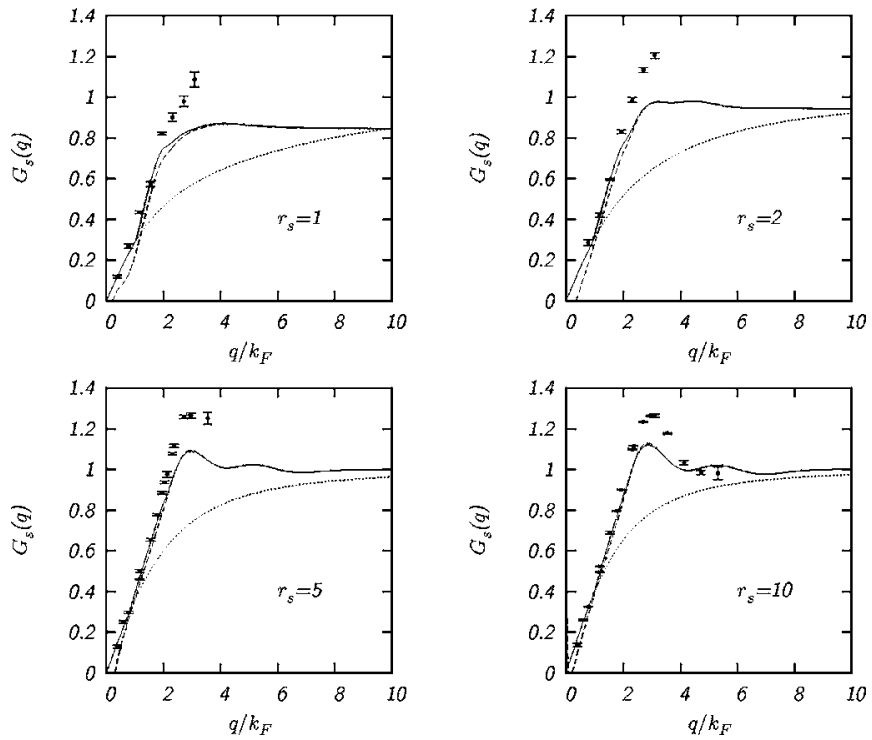

FIG. 2. The spin-symmetric local-field factor $G_{s}(q)$ for various values of $r_{s}$, in comparison with QMC data of Ref. 5. Solid, dotted, and dashed lines indicate using Eq. (9), polarization potential model, and MSA, respectively.

dure using $\chi_{0}^{\mathrm{MSA}}(q, \omega)$. These theoretical calculations are compared with the QMC data obtained from the static response calculations. ${ }^{5}$

There are several noteworthy points based on the results shown in Fig. 2. (i) The results of the procedure to extract $G_{s}(q)$ based on the fluctuation-dissipation theorem seem to be insensitive whether the full Lindhard $\chi_{0}(q, \omega)$ or the $\chi_{0}^{\mathrm{MSA}}(q, \omega)$ are used. (ii) The long-wavelength limit of $G_{s}(q)$ is not recovered in the fluctuation-dissipation theorem based calculations. Similar observations were also made by Iwamoto et al. ${ }^{16}$ in their calculation of the 3D $G_{s}(q)$. We thus correct our $G_{s}(q)$ by using the long-wavelength form of po-
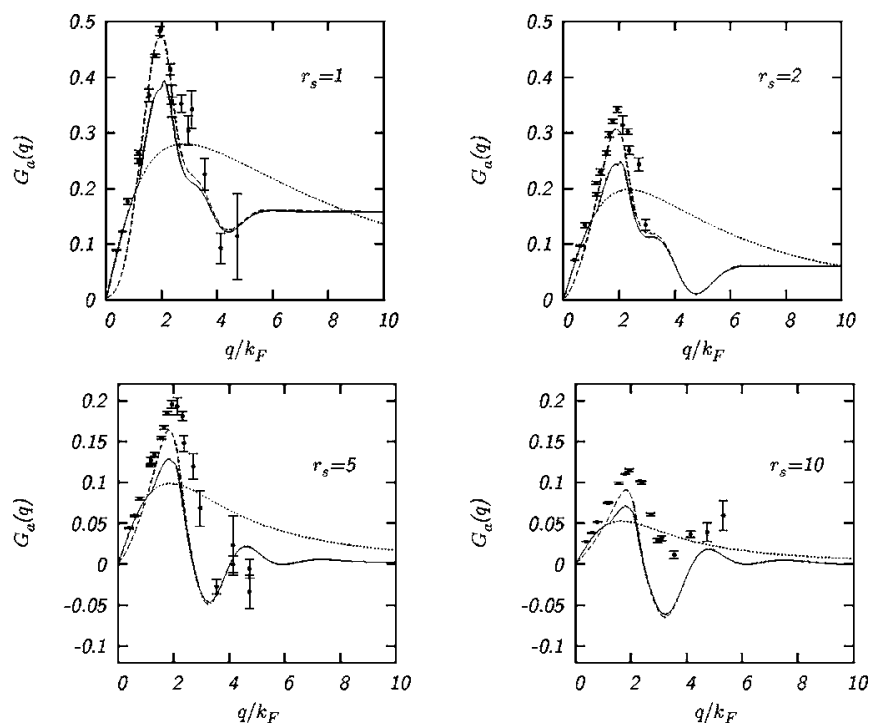

FIG. 3. The spin-antisymmetric local-field factor $G_{a}(q)$ for various values of $r_{s}$, in comparison with QMC data of Ref. 5. Solid, dotted, and dashed lines indicate using Eq. (9), polarization potential model, and MSA, respectively.
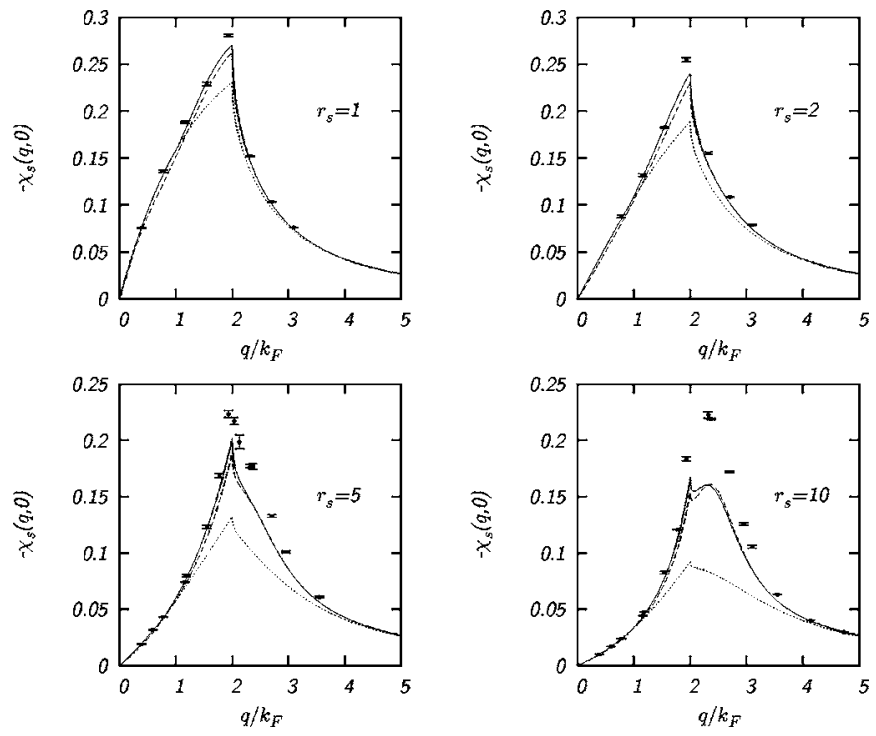

FIG. 4. The static spin-symmetric response function $\chi_{s}(q, 0)$ for various values of $r_{s}$, in comparison with QMC data of Ref. 5. Solid, dotted, and dashed lines indicate using Eq. (9), polarization potential model, and MSA, respectively.

larization potential calculation which is constructed to satisfy this limit. (iii) The polarization potential approach of Iwamoto gives the correct long-wavelength limit as it agrees quite well with the QMC data. For larger values of $q$, on the other hand, it fails to represent the peaked and oscillatory structures. (iv) The local-field factors we extract are qualitatively similar to those calculated by Dharma-wardana and Perrot $^{21,24}$ in the classical mapping hypernetted chain (CHNC) approximation. That is, a peak around $q \approx 3 k_{F}$ and subsequent oscillatory behavior at larger $q$ values, especially at large $r_{s}$. In fact, our fluctuation-dissipation theorem based procedure is quite similar to the way Dharma-wardana and
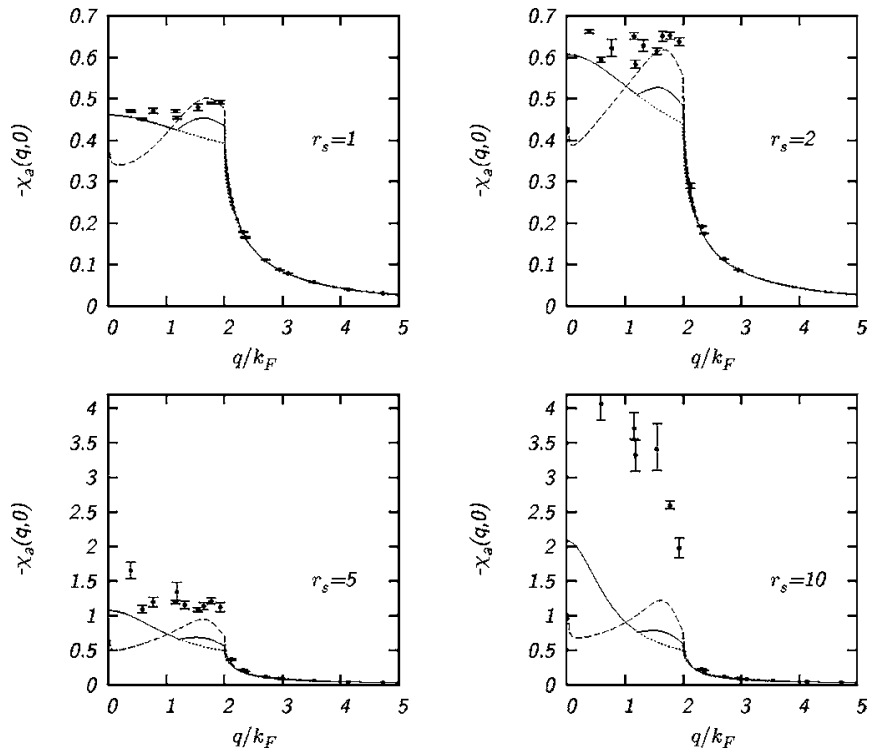

FIG. 5. The static spin-antisymmetric response function $\chi_{a}(q, 0)$ for various values of $r_{s}$, in comparison with QMC data of Ref. 5. Solid, dotted, and dashed lines indicate using Eq. (9), polarization potential model, and MSA, respectively. 
Perrot $^{21}$ obtain their local-field factors. In their case, the static structure factors from the CHNC calculation are used in conjunction with the fluctuation-dissipation theorem which for classical systems relates the static response function $\chi(q)$ to $S(q)$ directly without the frequency integral.

Figure 3 shows the similarly calculated spinantisymmetric local-field factor $G_{a}(q)$ at $r_{s}=1,2,5$, and 10 . First, $G_{a}(q)$ appears to have more structure than $G_{s}(q)$. Second, the oscillations in $G_{a}(q)$ at large $q$ and large $r_{s}$ are such that the local-field factor becomes negative. We again subtract the local field factor $G_{n}(q)$ as before. We also note that using the full $\chi_{0}(q, \omega)$ or $\chi_{0}^{\mathrm{MSA}}$ makes an important difference around the first peak of $G_{a}(q)$ which is at $q \approx 2 k_{F}$. The polarization potential approach constructed to yield the correct long-wavelength limit does not capture the oscillatory behavior.

We propose to modify the long-wavelength limit of our fluctuation-dissipation theorem extracted local-field factors by the polarization potential results since they are constructed to have the correct $q \rightarrow 0$ behavior. Furthermore, since we use the QMC static structure factors whose Fourier transforms are related to the pair correlation functions, the large $q$ limits of $G_{s}(q)$ and $G_{a}(q)$ are also fulfilled, i.e., $G_{s}(q \rightarrow \infty)=1-g(0)$ and $G_{a}(q \rightarrow \infty)=g(0)$. We thus have $G_{s}(q)$ and $G_{a}(q)$ representing the QMC results quite well in the whole range of $q$ values. Modifying the local-field factors by $G_{n}(q)$ is important to obtain the correct large $q$ limit as evidently reproduced by QMC results. These static localfield factors can now be used to calculate various other physical properties of a $2 \mathrm{D}$ electron liquid at large $r_{s}$ where there is recently so much interest.

We show in Fig. 4 the nonlocal susceptibility $\chi_{s}(q, 0)$ including $G_{s}(q)$ calculated by our procedure for $r_{s}=1,2,5$, and 10. Also shown for comparison are the QMC results ${ }^{5}$ which are calculated by the $G_{n}(q)$ contribution of $G_{s}^{\mathrm{QMC}}$ taken out, at the same $r_{s}$ values. We find good agreement with QMC results affirming the consistency and soundness of our procedure. The largest discrepancy at $r_{s}=10$ may be attributed to the approximate nature of $G_{n}(q)$ at these large values of $r_{s}$ as already indicated above.

Finally, Fig. 5 displays the nonlocal spin-susceptibility $\chi_{a}(q, 0)$ including $G_{a}(q)$ calculated by our procedure for $r_{s}=1,2,5$, and 10. We compare our results with those of QMC. In this case, too, the agreement is quite good except at $r_{s}=10$.

We note that the approach we take in this work does not rely on any interpolation of QMC data at low $r_{s}$ values as in the construction of Davoudi et al. ${ }^{13}$ We can obtain the localfield factors $G_{s, a}(q)$ once the corresponding static structure factors from QMC are known at a given $r_{s}$. Having compared our calculated static local-field factors with the QMC data in the range $1 \leqslant r_{s} \leqslant 10$, we now display $G_{s}(q)$ in Fig. 6 at $r_{s}=40$. The QMC data for the $r_{s}=40$ value is extracted

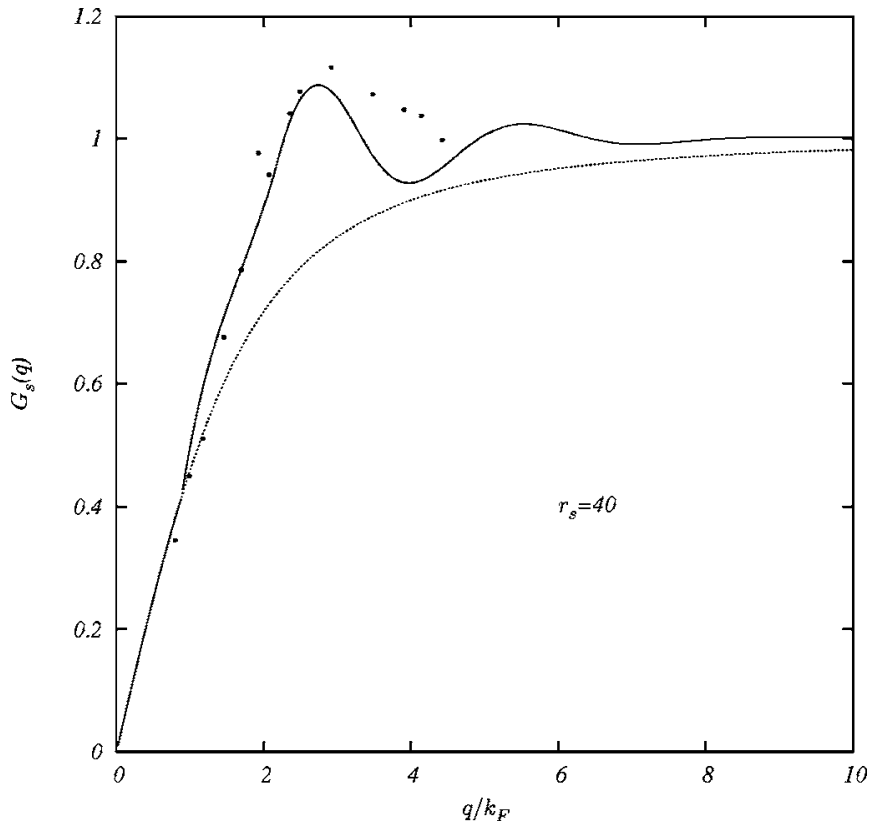

FIG. 6. The spin-symmetric local-field factor $G_{s}(q)$ (solid line) at $r_{s}=40$ compared to the QMC data (symbols) extracted from Ref. 14. Dotted line indicates the polarization potential model.

from the published figure in the work of Moreno and Marinescu. ${ }^{14}$ Atwal et al. ${ }^{11}$ parametrization of $G_{n}(q)$ does not work beyond $r_{s}>10$, thus we have not employed the subtraction procedure. It is seen that the fluctuation-dissipation theorem procedure yields a reasonable spin-symmetric localfield factor $G_{s}(q)$ in comparison to the QMC data even at this very large $r_{s}$ value.

\section{CONCLUSION}

In summary, we have presented in this work a numerical study of the spin-symmetric and spin-antisymmetric localfield factors for the 2D unpolarized electron liquid. Our approach which is based on a static approximation to the fluctuation-dissipation theorem yields numerical results of good quality if the small- $q$ regime is fitted to the compressibility and spin-susceptibility sum-rules (for spin-symmetric and spin-antisymmetric local-field factors, respectively) in the regime of weak and intermediate coupling strengths. As we have already commented in the main text, $G_{n}(q)$ may be treated more accurately in order to obtain better quantitative comparison with the $\mathrm{QMC}$ results.

\section{ACKNOWLEDGMENTS}

We thank S. Moroni for providing us with the QMC simulation data. R. A. would like to thank the Physics Department, Bilkent University at Bilkent, for its hospitality during the period when part of this work was carried out. A. L. S. thanks TUBITAK-BDP for support. B. T. acknowledges the support by TUBITAK and TUBA. 
${ }^{1}$ G. F. Giuliani and G. Vignale, Quantum Theory of the Electron Liquid (Cambridge University Press, Cambridge, 2005).

${ }^{2}$ E. Abrahams, S. V. Kravchenko, and M. P. Sarachik, Rev. Mod. Phys. 73, 251 (2001).

${ }^{3}$ B. Tanatar and D. M. Ceperley, Phys. Rev. B 39, 5005 (1989).

${ }^{4}$ C. Attaccalite, S. Moroni, P. Gori-Giorgi, and G. B. Bachelet, Phys. Rev. Lett. 88, 256601 (2002); 91, 109902(E) (2003).

${ }^{5}$ G. Senatore, S. Moroni, and D. M. Ceperley, in Quantum Monte Carlo Methods in Physics and Chemistry, edited by M. P. Nightingale and C. J. Umrigar (Kluwer, Dordrecht, 1999), p. 183; S. Moroni, D. M. Ceperley, and G. Senatore, Phys. Rev. Lett. 69, 1837 (1992); S. Moroni (private communication).

${ }^{6}$ P. Gori-Giorgi, S. Moroni, and G. B. Bachelet, Phys. Rev. B 70, 115102 (2004).

${ }^{7}$ G. Mahan, Many-Particle Physics (Plenum, New York, 1990).

${ }^{8}$ K. S. Singwi, M. P. Tosi, R. H. Land, and A. Sjölander, Phys. Rev. 176, 589 (1968).

${ }^{9}$ V. D. Gorobchenko, V. N. Kohn, and E. G. Maksimov, in The Dielectric Function in Condensed Systems, edited by L. V. Keldysh, D. A. Kirzhnitz, and A. A. Maradudin (North-Holland, Amsterdam, 1989), p. 87.

${ }^{10}$ R. Asgari, B. Davoudi, M. Polini, G. F. Giuliani, M. P. Tosi, and G. Vignale, Phys. Rev. B 71, 045323 (2005) R. Asgari, B. Davoudi, and B. Tanatar, Solid State Commun. 130, 13 (2004).
${ }^{11}$ G. S. Atwal, I. G. Khalil, and N. W. Ashcroft, Phys. Rev. B 67, 115107 (2003).

${ }^{12}$ Z. Qian and G. Vignale, Phys. Rev. B 65, 235121 (2002); 71, 169904(E) (2005).

${ }^{13}$ B. Davoudi, M. Polini, G. F. Giuliani, and M. P. Tosi, Phys. Rev. B 64, 153101 (2001); 64, 233110 (2001).

${ }^{14}$ J. Moreno and D. C. Marinescu, Phys. Rev. B 68, 195210 (2003).

${ }^{15}$ N. Iwamoto, Phys. Rev. B 43, 2174 (1991).

${ }^{16}$ N. Iwamoto, E. Krotscheck, and D. Pines, Phys. Rev. B 29, 3936 (1984).

${ }^{17}$ G. Senatore and G. Pastore, Phys. Rev. Lett. 64, 303 (1990).

${ }^{18}$ S. Hellal, J.-G. Gasser, and A. Issolah, Phys. Rev. B 68, 094204 (2003).

${ }^{19}$ N. Iwamoto and D. Pines, Phys. Rev. B 29, 3924 (1984).

${ }^{20}$ V. Apaja, J. Halinen, V. Halonen, E. Krotscheck, and M. Saarela, Phys. Rev. B 55, 12925 (1997).

${ }^{21}$ M. W. C. Dharma-wardana and F. Perrot, Europhys. Lett. 63, 660 (2003).

${ }^{22}$ Some expressions in Ref. 11 are corrected by R. Asgari, M. Polini, B. Davoudi, and M. P. Tosi, Phys. Rev. B 68, 235116 (2003).

${ }^{23}$ A. Holas, in Strongly Coupled Plasma Physics, edited by F. J. Rogers and H. E. Dewitt (Plenum, New York, 1987), p. 463.

${ }^{24}$ F. Perrot and M. W. C. Dharma-wardana, Phys. Rev. B 62, 16536 (2000). 